\title{
Various statistical methods in use for evaluating human malignant gastric specimens*
}

\author{
Ventzeslav Enchev ${ }^{\mathrm{a}, * *}$ and Mircho Vukov \\ ${ }^{a}$ Department of Pathology, Third City Hospital, Sofia, Bulgaria \\ ${ }^{\mathrm{b}}$ National Centre of Health Informatics, Sofia, Bulgaria
}

Received 2 June 1997

Revised 24 November 1997

Accepted 2 January 1998

This paper is dedicated to †Ing. George Enchev, the father of the first author, who died on 11.10.1997.

\begin{abstract}
This paper presents the use of certain statistical methods (comparison of means - independent samples $t$-test, multiple linear regression analysis, multiple logistic regression analysis, analysis of clusters, etc.) included in the SPSS Statistical Package used to classify the patients quantitatively evaluated after a subtotal resection of their stomachs. The group consisted of 40 patients subdivided into two groups: primary neoplasia of the stomach (20 patients), and corresponding lymphogenic deposits in the abdominal perigastric lymph nodes (20 patients). Paraffin-embedded tissue sections (thickness 4-5 $\mu \mathrm{m})$ prepared as consecutive hematoxylin-eosin-stained slides were morphometrically measured by a rotation of a graduated eyepiece-micrometer; thus, we obtained the minor and major axes' lengths of the elliptic nuclear profiles and the minor and major caliper diameters of the corresponding cellular profiles. These four variables were used to determine the dynamic changes in quantitative features of human gastric lesions when passing from normal histological structures, through hyperplastic processes (chronic gastritis), gastric precancer (ulcers and polyps with or without malignancy) till the development of primary carcinomas and their corresponding lymphogeneous metastases. Besides the increased cytomorphometrical measures, we also noted an opportunity to classify the patients according to these data as well as to add to the knowledge of our consultation system for clinical aid and use, recently published in the literature.
\end{abstract}

Keywords: Human gastric specimens, cytomorphometry, statistics

\section{Introduction}

The question of tumour progression, i.e., how and when primary tumours will spread and give earlier or later metastases, is of current interest. The locations of distant secondary tumours in many clinical and animal tumours are nonrandom and their distributions cannot be explained by simple

${ }^{*}$ This paper was presented at 5th Congress of European Society for Analytical and Cellular Pathology, May 25-29, 1997, Oslo, Norway.

** Corresponding author: Dr. Ventzeslav Enchev, M.D., Ph.D., 6, Visheva street, 1164 Sofia, Bulgaria. 
anatomical or mechanical hypotheses based on the simple trapping of tumour cell emboli in the first capillary bed encountered. Evidence from certain experimental tumour systems supports Paget's "seed and soil" hypothesis on the nonrandom distributions of metastases in which the unique properties of particular tumour cells ("seeds") and the different characteristics of each organ microenvironment ("soil") collectively determine the organ preference of metastases. Each tumour system may achieve organ specificity because of its own unique set of multiple associated properties and responses to

host microenvironments. According to Paget's hypotheses tumour cells mechanically lodge in the first capillary bed encountered during their circulation in the blood, and this is the most important consideration in determining the secondary locations of neoplasms. Indeed, in most cancers where multicellular tumour emboli are released into the lymph or circulation, these emboli are not expected to easily traverse small nodes or vessels. Regional metastatic involvement can be explained, for the most part, by different venous circulation or lymphatic drainage patterns [9].

The lung is an expected frequent site for distant metastatic colonization, because passive mechanical location of circulating tumour emboli should occur here first. Moreover, the lung and liver [11] are the central organs for the settlement of metastatic tumour cells escaping from malignancies located in various organs. Another paper [8] noted that the ability of tumour cells to metastasize is now understood as a combination of three different phenomena as follows:

(i) It is dependent on the number of malignant cells and, therefore, a stochastic process. Tumour cells have to escape from the bulk tumour and migrate into the lymphatic and blood vessels.

(ii) It is dependent upon the location of the primary tumour with respect to the corresponding organ of metastases, i.e., a phenomenon of topography.

(iii) The microenvironment plays a predominant role. The ability of tumour cells to grow in an environment different from the primary tumour organ is a prerequisite for metastatic tumour growth [8].

Moreover, primary tumours with large nuclear areas are more liable to invade lymph nodes [14]; a high resolution image analysis of cell nuclei might be used for histological typing of primary and metastatic carcinomas of oropharynx, larynx, oesophagus, gastrointestinal tract and infiltrative ductal carcinomas of the breast [12,13]; karyometric criteria may help in improving the reproducibility of gradings [10].

The aim of this paper is to present the evaluation of the interdependences between the primary gastric tumours and their corresponding perigastric lymph node deposits in the abdominal region by using the SPSS Statistical Package [7]; thus, we can add to the knowledge of our consultation system recently published elsewhere [3]. These efforts are based on our previous publication [2] where we noted lesser quantitative cytomorphometrical variables in the patients with regional perigastric lymph node metastases.

\section{Materials and methods}

\subsection{Specimen preparation and morphometrical assessments $[4,6]$}

We examined 20 patients with primary gastric carcinomas and 20 with corresponding perigastric lymph node metastases; within the group 10 patients had only primary or only metastatic tumour nodes and 10 had both processes together. Biopsies were obtained after a subtotal resection of their stomachs 
and were prepared as consecutive hematoxylin-eosin-stained slides. Paraffin-embedded tissue sections with a thickness of $4-5 \mu \mathrm{m}$ were routinely diagnosed.

Cytomorphometric measurements were carried out by rotation of a graduated eyepiece-micrometer and standard optical conditions on an "Amplival" microscope (Carl-Zeiss, Jena) as follows: objective $100: 1 \times$, ocular $10: 1 \times$ and oil immersion with refractive index at room temperature 1.515 . One hundred randomly chosen nonmitotic nuclei and corresponding cells were measured in each case; thus, the following quantitative variables were obtained: minor $\left(B_{\mathrm{N}}\right)$ and major $\left(L_{\mathrm{N}}\right)$ nuclear axes' lengths, and minor $\left(B_{\mathrm{c}}\right)$ and major $\left(L_{\mathrm{c}}\right)$ cellular caliper diameters.

\subsection{Statistical processing and interpretation of the data}

Classical statistical parameters of $t$-test were obtained by using a program written by Kalpasanov and Draganov for DISNOR NOVA 840 minicomputer as follows: arithmetical mean; standard deviation; Pearson's coefficient of variation; and error of representation [6].

We also used the SPSS Statistical Package (comparison of means, independent samples $t$-test, multiple linear regression analysis, multiple logistic regression analysis, cluster analysis, etc.). The multiple logistic regression analysis was used to classify patients into groups [1]. $L_{\mathrm{c}}$ was accepted as a dependent variable compared with the other independent quantitative features; this variable was selected as a sign of the final stage of the mitotic cycle after the changes during nuclear mitosis. We used the stepwise method in the multiple regression analyses.

The data obtained were graphically presented as predicted, and obtained values and were put into multiple linear regression equations.

\section{Results}

\subsection{Primary carcinomas of the stomach}

Our data showed that $L_{\mathrm{c}}$ as a dependent feature compared with the other three independent quantitative variables showed dependences presented in the equation as follows:

$$
L_{\mathrm{c}}=5.261+0.954 B_{\mathrm{c}} .
$$

The $B_{\mathrm{c}}$ coefficient is significant $(p<0.0001)$.

The other two independent quantitative variables were excluded from the multiple regression equation.

\subsection{Corresponding metastases in perigastric lymph nodes}

Our data showed that metastatic gastric carcinomas had different interdependences between the cytomorphometric variables: $L_{\mathrm{c}}$ as a dependent feature included two of the other variables such as $B_{\mathrm{c}}$ and $B_{\mathrm{N}}$. The multiple regression equation is as follows:

$$
L_{\mathrm{c}}=1.023+0.719 B_{\mathrm{c}}+0.437 B_{\mathrm{N}} .
$$

The $B_{\mathrm{c}}$ and $B_{\mathrm{N}}$ coefficients are significant $(p<0.0001, p<0.01)$. 
Table 1

Classification table for groups 1 and 2

\begin{tabular}{crcc}
\hline Observed & \multicolumn{2}{c}{ Predicted } & Percent correct (\%) \\
\cline { 2 - 3 } & 1 & 2 & \\
\hline 1 & 19 & 1 & 95.0 \\
2 & 0 & 20 & 100.0 \\
& & Overall & 97.5 \\
\hline
\end{tabular}

1 is the group of patients with primary carcinomas of the stomach; 2 is the group of patients with corresponding perigastric lymph node metastases.

Table 2

Table for the predicted probabilities as well as the observed and predicted groups

\begin{tabular}{|c|c|c|c|}
\hline Number of patient & Predicted probability & Observed group & Predicted group \\
\hline 1 & 0.03627 & 1 & 1 \\
\hline 2 & 0.00058 & 1 & 1 \\
\hline 3 & 0.00538 & 1 & 1 \\
\hline 4 & 0.04859 & 1 & 1 \\
\hline 5 & 0.02876 & 1 & 1 \\
\hline 6 & 0.91597 & 2 & 1 \\
\hline 7 & 0.00612 & 1 & 1 \\
\hline 8 & 0.01583 & 1 & 1 \\
\hline 9 & 0.00341 & 1 & 1 \\
\hline 10 & 0.00015 & 1 & 1 \\
\hline 11 & 0.02816 & 1 & 1 \\
\hline 12 & 0.00181 & 1 & 1 \\
\hline 13 & 0.00085 & 1 & 1 \\
\hline 14 & 0.00004 & 1 & 1 \\
\hline 15 & 0.00105 & 1 & 1 \\
\hline 16 & 0.00096 & 1 & 1 \\
\hline 17 & 0.00021 & 1 & 1 \\
\hline 18 & 0.00131 & 1 & 1 \\
\hline 19 & 0.00007 & 1 & 1 \\
\hline 20 & 0.00128 & 1 & 1 \\
\hline 21 & 0.95723 & 2 & 2 \\
\hline 22 & 0.99888 & 2 & 2 \\
\hline 23 & 0.99989 & 2 & 2 \\
\hline 24 & 0.95447 & 2 & 2 \\
\hline 25 & 0.99760 & 2 & 2 \\
\hline 26 & 0.81020 & 2 & 2 \\
\hline 27 & 0.90534 & 2 & 2 \\
\hline 28 & 0.99943 & 2 & 2 \\
\hline 29 & 0.99996 & 2 & 2 \\
\hline 30 & 0.99975 & 2 & 2 \\
\hline 31 & 0.99834 & 2 & 2 \\
\hline 32 & 0.96675 & 2 & 2 \\
\hline 33 & 0.99952 & 2 & 2 \\
\hline 34 & 0.99983 & 2 & 2 \\
\hline 35 & 0.98353 & 2 & 2 \\
\hline 36 & 0.93795 & 2 & 2 \\
\hline 37 & 0.55196 & 2 & 2 \\
\hline 38 & 0.99985 & 2 & 2 \\
\hline 39 & 0.84437 & 2 & 2 \\
\hline 40 & 0.99834 & 2 & 2 \\
\hline
\end{tabular}




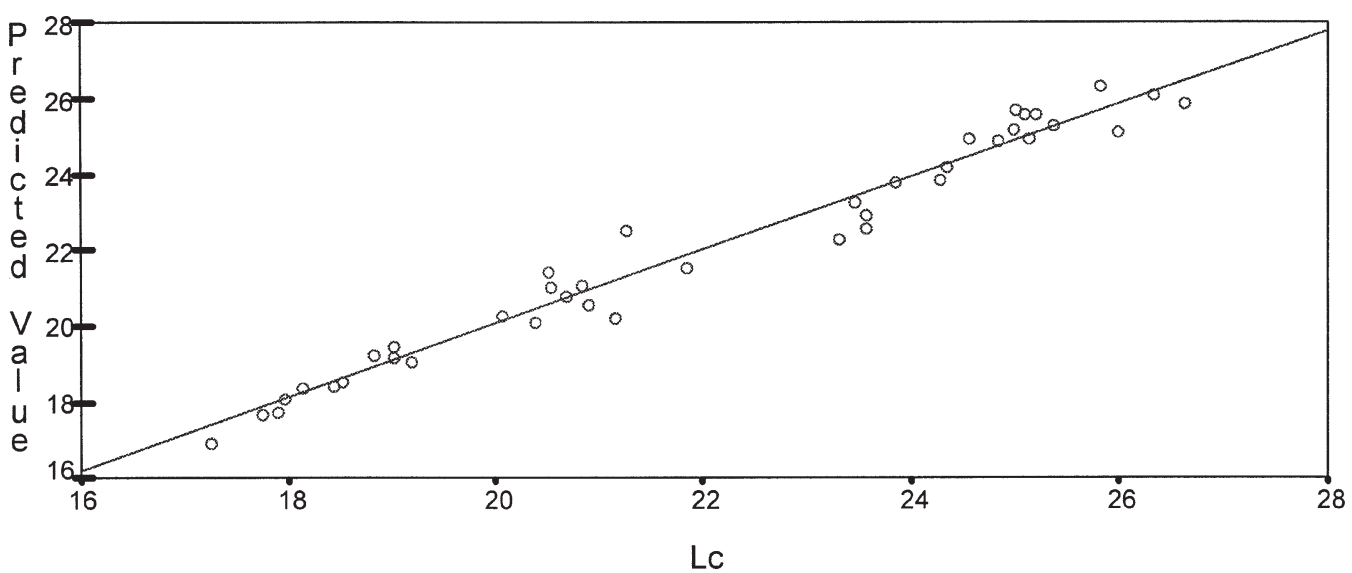

Fig. 1. Scattergraph on values obtained and predicted of $L_{\mathrm{c}}$ in case with primary carcinomas of the stomach and the corresponding metastasis.

The stepwise multiple logistic regression analysis showed that the use of only one variable, i.e., $L_{\mathrm{c}}$, is enough to differentiate primary tumours from their corresponding metastatic deposit.

$$
P_{\text {meta }}=\frac{1}{1+\mathrm{e}^{47.8458-2.1802 L_{\mathrm{c}}}} ;
$$

$P_{\text {meta }}$ is the probability that the patient be included in the group of those with lymph node metastases. The $L_{\mathrm{c}}$ coefficient is significant $(p=0.02)$. The predicted probabilities as well as the observed and predicted groups are shown in Table 2; Table 1 presents a classification of groups 1 and 2 .

The multiple regression equation of the summarized groups of patients (40 patients with primary carcinomas of the stomach mucosae and the corresponding metastases in perigastric lymph nodes) was obtained as follows:

$$
L_{\mathrm{c}}=-0.844146+1.098318 B_{\mathrm{c}}-1.074976 B_{\mathrm{N}}+1.060522 L_{\mathrm{N}} .
$$

The $B_{\mathrm{c}}, B_{\mathrm{N}}$ and $L_{\mathrm{N}}$ coefficients are significant $(p<0.0001)$.

The scattergraph on values obtained and predicted of $L_{\mathrm{c}}$ in cases with primary carcinomas of the stomach and the corresponding metastases in perigastric lymph nodes are shown in Fig. 1.

Data of $L_{\mathrm{c}}$ from randomly chosen patients may predict the probability of their belonging to the groups of primary blastomas or corresponding lymphatic deposits. The equation showed the probability that the patients belong to the group of metastases.

The probability that the patients belong to the group of primary carcinomas is shown as follows: $P_{\text {carcinoma }}=1-P_{\text {meta }}$.

\section{Discussion}

These results, based on the use of various statistical methods after the quantitative cytomorphometric measurements, confirmed on the one hand the results of these measurements and, on the other hand, noted the usefulness of the introduction of these methods. In this study we chose the major cellular caliper diameter $\left(L_{\mathrm{c}}\right)$ as a dependent variable because this feature was larger than the other three quantities. Moreover, during the mitotic cycle, cells divide after the division of the nuclei; thus, 
predicted and obtained values on $L_{\mathrm{c}}$ in both groups of patients as well as linear regression equations might be valid. Our data showed that in cases of primary carcinomas of the stomach only $B_{\mathrm{c}}$ was included in the regression equation. Furthermore, in cases with corresponding deposits in the perigastric lymph nodes in the abdomen, $B_{\mathrm{N}}$ was included as well as $B_{\mathrm{c}}$. The stepwise multiple logistic regression analysis showed us that the use of only one of these 4 quantities, the major cellular caliper diameter $\left(L_{\mathrm{c}}\right)$, is enough to differentiate the patients with primary tumours from those with the corresponding metastases in the abdominal region.

Another paper [2] noted that the parameters obtained by the cytomorphometric measurements were found to be inferior to cases with corresponding metastases in perigastric lymph nodes in the abdomen compared with patients who had primary carcinomas of the stomach. A possible interpretation is that clones of a particular slightly hyperdiploid cell population would be selected at the onset of invasive cancer and again later, when metastases occurs.

In conclusion, we can assume that all these data from previous and recent studies give us additional valuable information about the dynamic changes in gastric mucosae upon passage from the norm through the various steps of their malignant transformation.

\section{References}

[1] D.G. Altmann, Practical Statistics for Medical Research, Chapman and Hall, 1991, p. 591.

[2] V. Enchev, Gastric carcinomas and their corresponding lymphogeneous metastases in cytomorphometric expression, Gegenbaurs. Morph. Jahrb. (Leipzig) 130 (1984), 565-571.

[3] V. Enchev, J. Angelova and J. Peneva, A consultation system for diagnosis of gastric lesions, Anal. Quant. Cytol. Histol. 17 (1997), 13-18.

[4] V. Enchev and R.D. Raichev, Zytomorphometrische Untersuchungen bei normaler Magenschleimhaut, präkanzerösen Krankheiten und Magenkrebs, Arch. Geschwulstforsch. 52 (1982), 641-647.

[5] V. Enchev and J.-P. Rigaut, Dynamic changes in quantitative features in human gastric lesions, Anal. Cell. Pathol. 13 (1997), 9-16.

[6] V. Enchev and K.G. Tsanev, Comparative cytomorphometric and cytospectrophotometric investigations of gastric lesions, Arch. Geschwulstforsch. 55 (1985), 37-46.

[7] V. Enchev, M. Vukov and S. Gospodinova, Statistical analysis of human gastric biopsies, Anal. Quant. Cytol. Histol., in press.

[8] K. Kayser, Metastases into the lung - the pathologist's point of view, Contr. Oncol. 30 (1988), 19-28.

[9] G.L. Nicolson, Organ specificity of tumour metastases: role of preferential adhesion, invasion and growth of malignant cells at specific sites, Cancer Metastas. Rev. 7 (1988), 143-188.

[10] J.P. Rigaut, Karyometry on sections from dysplastic and cancerous tissues, in: Quantitative Image Analysis in Cancer Cytology and Histology, J.Y. Mary and J.P. Rigaut, eds, Elsevier, Amsterdam, 1986, pp. 207-222.

[11] N.S.E. Sargent, M. Oestreicher, H. Haidvogl, H.M. Maduick and M.M. Burger, Growth regulation of cancer metastases by their host organ, Proc. Natl. Acad. Sci. USA 85 (1988), 7251-7255.

[12] F. Theissig, V. Dimmer and K.D. Kunze, High resolution image analysis of cell nuclei in tissue sections of primary and metastatic carcinomas, Arch. Geschwulstforsch. 56 (1986), 241-247.

[13] F. Theissig, K.D. Kunze and V. Dimmer, High resolution image analysis of cell nuclei in tissue sections for histological typing of primary and metastatic carcinomas, in: Automated Image Analysis in Medicine and Biology. Proceedings, W. Herrmann, K.D. Kunze and V. Dimmer, eds, Johann Ambrosius Barth, Leipzig, 1988, pp. 192-208.

[14] P. Tosi, P. Luzi, V. Sforza, D. Spina, C. Miracco and R. Santopietro, Morphometrical assessment of mean nuclear area of lymph node metastases, Pathol. Res. Pract. 180 (1985), 498-501.

[15] H.C. Vanderlinden, J.P.A. Baak, J. Lindeman, J. Hermans and C.J.L.M. Meyer, Morphometry and breast cancer II. Characterization of breast cancer cells with high malignant potential in patients with spread to lymph nodes: preliminary results, J. Clin. Pathol. 39 (1986), 603-609.

[16] H.C. Vanderlinden, J.P.A. Baak, A.W.M. Smeulders, J. Lindeman and C.J.L.M. Meyer, Morphometry and breast cancer I. Comparison of the primary tumours and the axillary lymph node metastases, Pathol. Res. Pract. 181 (1986), $236-242$.

[17] A. Zaidela, B. Asselain and N.A. Ghossein, Comparison between the nuclear diameters of primary and metastatic breast cancer cells obtained by cytological aspiration, Cancer 56 (1985), 1605-1610. 


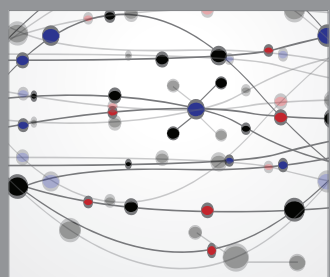

The Scientific World Journal
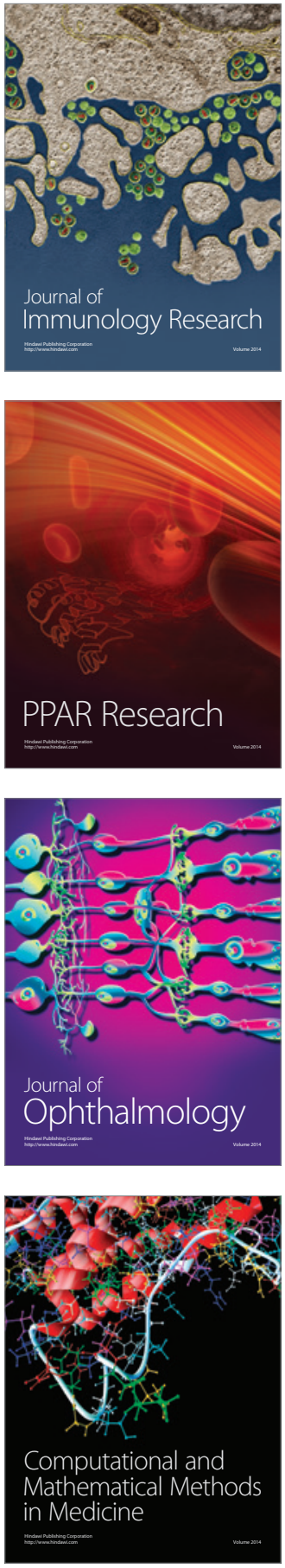

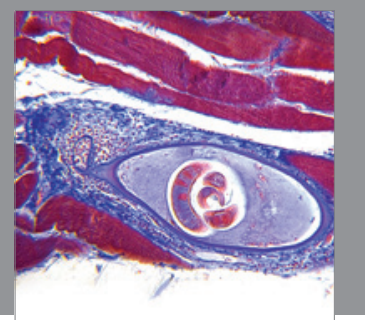

Gastroenterology

Research and Practice
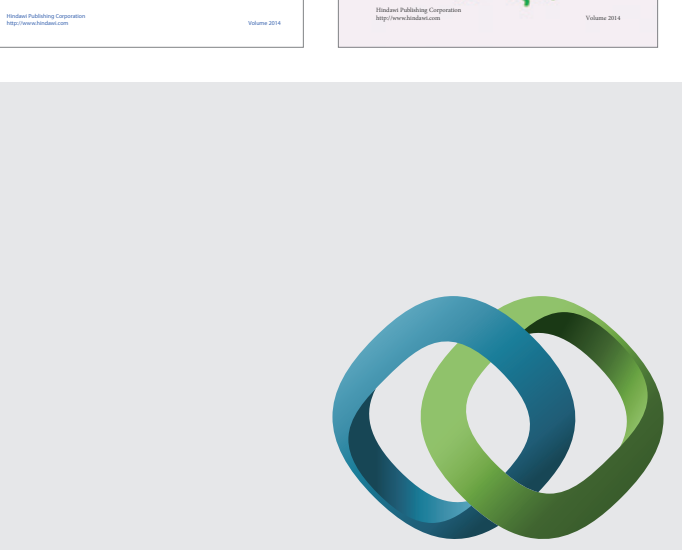

\section{Hindawi}

Submit your manuscripts at

http://www.hindawi.com
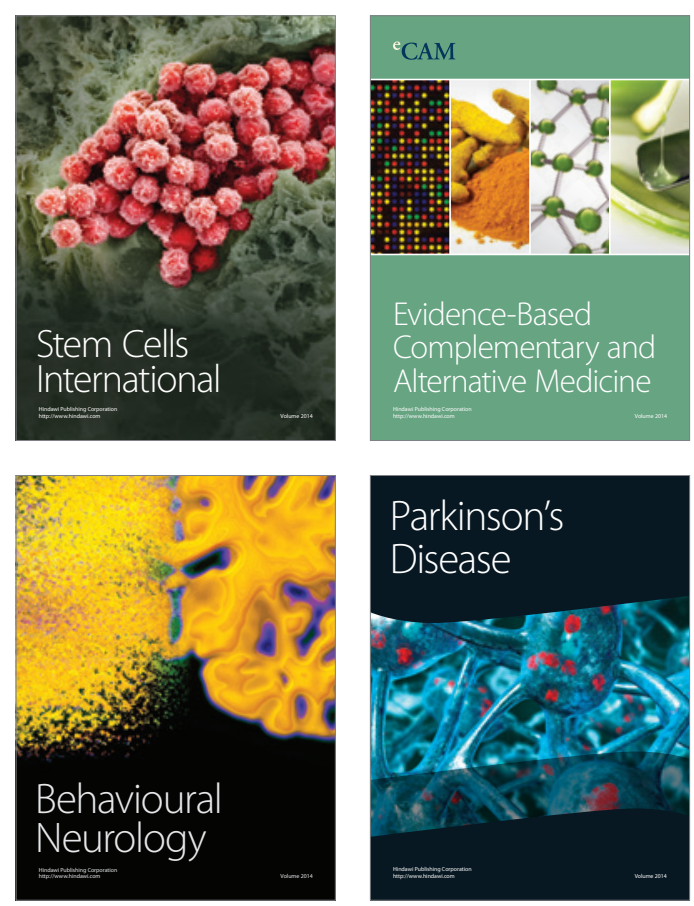

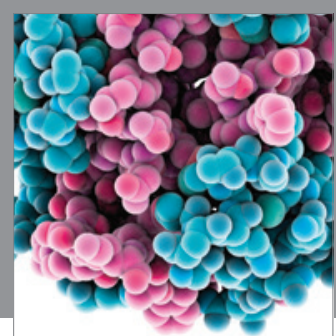

Journal of
Diabetes Research

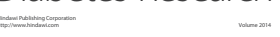

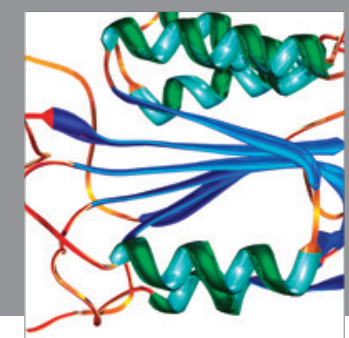

Disease Markers
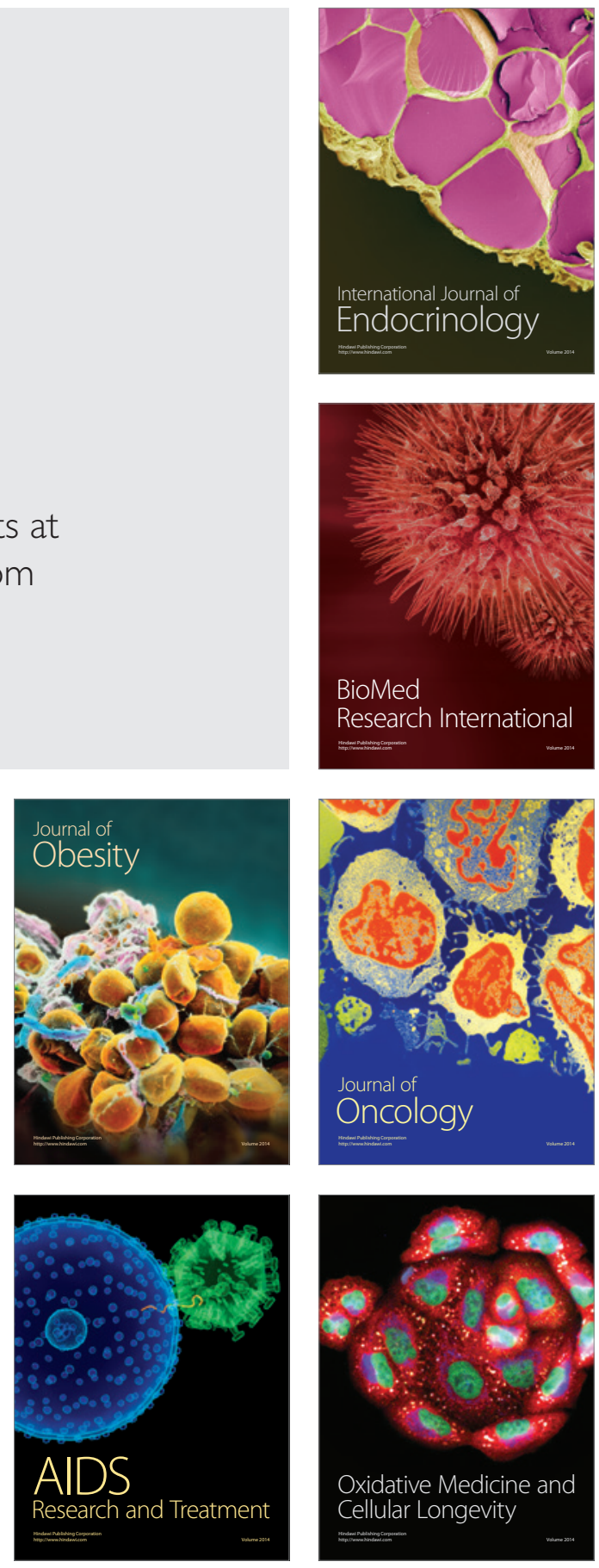